\title{
Supporting Configurable Congestion Control in Data Transport Services
}

\author{
Yunhong $\mathrm{Gu}^{1}$ and Robert L. Grossman ${ }^{1,2}$ \\ 1. University of Illinois at Chicago \\ 2. Open Data Partners \\ \{ygu3, grossman\}@uic.edu
}

\begin{abstract}
As wide area high-speed networks rapidly increase, new applications emerge and require new control mechanisms in data transport services to support them. In this paper, we present UDT/CCC, a data transport library that allows users to make use of a new control algorithm through simple configurations. We aim to provide a tool for fast implementation and deployment, as well as easy evaluation, of new congestion control algorithms. UDT/CCC uses an objected-oriented design. We show that our UDT/CCC library can be used to easily implement a large variety of control algorithms and can simulate the behavior of their native implementations as well. The UDT/CCC library is at the application level and it does not need root privilege to be installed. Meanwhile, it was specially developed to require very few changes to the existing applications. This paper describes its design, implementation, and evaluation.
\end{abstract}

\section{Categories and Subject Descriptors}

C.2.2 [Network Protocols]: Protocol Architecture

\section{General Terms}

Management, Performance, Design, Experimentation

\section{Keywords}

Transport Protocol, Congestion Control, Protocol Framework.

\section{INTRODUCTION}

The rapid increase of network bandwidth has enabled numerous distributed data intensive applications. These new applications vary from bulk data transfer (e.g., SDSS [34] and e-VLBI [40]) to high throughput interactive systems (e.g., GeoWall [35]). Different applications have specific requirements for data transfer services. For example, a GeoWall application may prefer a smooth data transfer rate, whereas it is desirable to move SDSS data at the highest possible speed in private networks.

Permission to make digital or hard copies of all or part of this work for personal or classroom use is granted without fee provided that copies are not made or distributed for profit or commercial advantage, and that copies bear this notice and the full citation on the first page. To copy otherwise, to republish, to post on servers or to redistribute to lists, requires prior specific permission and/or a fee.

SC|05 November 12-18, 2005, Seattle, Washington, USA

(c) 2005 ACM 1-59593-061-2/05/0011 ..\$5.00
However, the current Internet is designed to provide general service to support as many different applications as possible. This design philosophy has a major impact on the transport protocols. The majority of traffic on the Internet is contributed by TCP flows; but there still are applications that TCP cannot support well. In the context of high performance computing, TCP is well known for its poor efficiency and fairness in high bandwidth delay product networks $[1,2]$.

In the past few years, network researchers have proposed numerous new congestion control algorithms for both general and specific data transfers. However, most of them only end with simulations and limited laboratory experiments; few get tested and deployed in real networks. On the one hand, modifications of the kernel network stack (e.g., new TCP variants) usually take years for standardization, implementation, and widespread deployment. On the other hand, although user space stacks are much easier to get deployed, it is often a painstaking and timeconsuming job to implement each of them from scratch. In fact, ever since the emergence of TCP about three decades ago, only four versions have been widely deployed, namely Tahoe, Reno, NewReno, and SACK.

It is very desirable to create a configurable or reusable user space network stack on which a new congestion control algorithm can be easily implemented, deployed, and evaluated. First, a user space stack is much easier to get deployed, and so is the congestion control algorithms built in it. Second, this stack is useful to support application aware control approaches. An application may prefer to use different congestion control strategies in different situations. Third, this stack can save significant time for network researchers and developers because they can focus on the control algorithm itself rather than the whole protocol implementation. As a sequence, as there are more and more users, this stack can provide good software quality to support application development.

However, this stack is not a replacement for, but a complement to the kernel space network stacks. General protocols like UDP, TCP, DCCP [8], and SCTP [7] should still exist inside the kernel space of operating systems, but OS vendors may be reluctant to support too many protocols and algorithms, especially those application specific or network specific ones.

To address the above requirement, we developed the UDT/CCC library, or UDP-based Data Transfer library with Configurable Congestion Control. This work is based on our UDT library [1], which is a user space data transport library developed for high performance data intensive applications. CCC is one of the features included in the current UDT release. 
$\mathrm{UDT} / \mathrm{CCC}$ is written in $\mathrm{C}++$ and it provides a set of control events handlers and parameters in a base $\mathrm{C}++$ class. A new control algorithm inherits this base class, redefines the proper control event handlers, and modifies certain control parameters when necessary.

UDT/CCC supports a wide variety of control algorithms, including but not limited to, TCP algorithms (e.g., NewReno, Vegas [25], FAST [26], Westwood [24], HighSpeed [27], BiC [29], and Scalable [28]), bulk data transfer algorithms (e.g., SABUL [30], RBUDP [31], LambdaStream [32], CHEETAH [33], and Hurricane [41]), and group transport control algorithms (e.g., CM [5] and GTP [36]).

The UDT library provides a socket-like API so that applications can change their data transport service between TCP and UDT/CCC with ease. In certain situations, an existing TCP-based application can make use of UDT without any change to the source code.

We envision the following use scenarios for UDT/CCC:

- Implementation and deployment of new control algorithms. Certain control algorithms may not be appropriate to be deployed in kernel space, e.g., a bulk data transfer mechanism used only in private links. These algorithms can be implemented using UDT/CCC.

- Application awareness support and dynamic configuration. An application may choose different congestion control strategies under different networks, different users, and even different time slots. Furthermore, in certain circumstances, application level congestion/flow may be more preferable than kernel level control because the former can consider the specific requirements of host applications and information obtained from them. UDT/CCC supports these application aware algorithms.

- Evaluation of new control algorithms. Even if a control algorithm is to be deployed in kernel space, it needs to be tested thoroughly before OS vendors distribute the new version. It is much easier to test the new algorithms using UDT/CCC than modifying an OS kernel.

In this paper, we present the design, implementation, and evaluation of the UDT/CCC library. The rest of the paper is organized as follows. We begin with the CCC design and architecture in Section 2, followed by the key implementation details in Section 3. We then evaluate the UDT/CCC library in the following section. In the first part of Section 4, we demonstrate the expressiveness and simplicity of using UDT/CCC to develop new control algorithms. Then in the second part, we use experimental studies to examine the performance characteristics. Finally, we give a brief review of related work in Section 5 and conclude the paper in Section 6.

\section{ARCHITECTURE AND DESIGN}

The UDT/CCC library can be regarded as a middleware layer between applications and the network transport layer. The library is completely implemented at the application level above UDP. In this section, we focus on how the congestion control interface is provided by the library and how it is implemented inside the UDT layer.

\subsection{Overview}

The UDT framework has a layered architecture (Figure 1). UDT uses UDP through the socket interface provided by operating systems. Meanwhile, it provides a UDT socket interface to applications.

Applications can call the UDT sockets API in the same way they call the system sockets API. They can provide a congestion control class instance ( $\mathrm{CC}$ in Figure 1) for UDT to process the control events, or use the default congestion control algorithm [1] provided by UDT. The CC instance includes a set of necessary user-defined callback functions (control event handlers) to process certain control events.

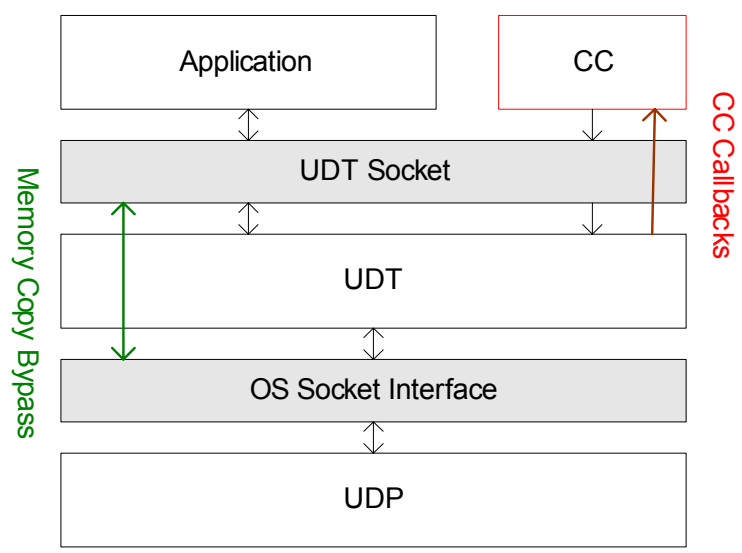

Figure 1. UDT/CCC Architecture. In this layered architecture, the UDT layer is completely in user space above the network transport layer of UDP, whereas the UDT layer itself provides transport for applications. Meanwhile, applications provide optional control handlers to UDT as callbacks.

\subsection{The CCC Interface}

We identify four categories of configuration features to support configurable congestion control mechanisms. They are 1) control event handler callbacks, 2) protocol behavior configuration, 3) packet extension and 4) performance monitoring.

\subsubsection{Control Event Callbacks}

Seven basic callback functions are defined in the base CCC class. They are called by UDT when a control event is triggered.

init and close: These two methods are called when a UDT connection is set up and when it is torn down. They can be used to initialize necessary data structures and release them later.

onACK: This handler is called when an ACK (acknowledgment) is received at the sender side. The sequence number of the acknowledged packet can be learned from the parameters of this method.

onLoss: This handler is called when the sender detects a packet loss event. The explicit loss information is given to users as the onLoss interface parameters. Note that this method may be redundant for most TCP algorithms that use only duplicate ACKs to detect packet loss. 
UDT generates negative acknowledgement (NAK) to report explicit loss information. This is equivalent to the selective acknowledgement mechanism.

onTimeout: A timeout event can trigger the action defined by this handler. The timeout value can be assigned by users, otherwise it uses the default value according to the TCP RTO (Retransmission Timeout) calculation described in RFC 2988 [11].

onPktSent: This is called right before a data packet is sent. The packet information (sequence number, timestamp, size, etc.) is available through the parameters of this method.

onPktReceived: This is called right after a data packet is received. Similar to onPktSent, the entire packet information can be accessed by users through the function parameters.

onPktSent and onPktReceived are the two most powerful event handlers, because they allow users to check every single data packet. For example, onPktReceived can be redefined to compute the loss rate in TFRC. Due to the same reason, these two callbacks can also allow users to trace the microscopic behavior of a protocol.

processCustomMsg: This method is used for UDT to process user-defined control messages.

\subsubsection{Protocol Configuration}

To accommodate certain control algorithms, some of the protocol behavior has to be customized. For example, a control algorithm may be sensitive to the way that data packets are acknowledged. UDT/CCC provides necessary protocol configuration APIs for these purposes.

It allows users to define how to acknowledge received packets at the receiver side. The functions of setACKTimer and setACKInterval determine how often an acknowledgement is sent, in elapsed time and number of arrived packets, respectively.

The method of sendCustomMsg sends out a user-defined control packet to the peer side of a UDT connection, where it is processed by callback functions processCustomMsg. This user-defined control packet can be used to support protocols like TFRC that need to general their own control packets.

Finally, UDT/CCC also allows users to modify the values of RTT (Round Trip Time) and RTO. A new congestion control class can choose to use either the RTT value provided by UDT, or its own calculated value. Similarly, the RTO value can also be redefined.

There are other features of the UDT protocol that are either not related to congestion control or are helpful to most control algorithms. These features, such as selective acknowledgement (SACK) [37] and robust reordering (RR) [38], cannot be configured by CCC users, although some of the features can be configured through UDT interfaces.

\subsubsection{Packet Extension}

It is necessary to allow user-defined control packets for a configurable protocol stack.

Because our UDT/CCC library is mainly focused on congestion control algorithms, we only give limited customization ability to the control packets. Data packet processing contributes to a large portion of CPU utilization and customized data packets may hurt the performance.
A UDT data packet contains a packet-based sequence number and a relative timestamp (it starts counting since the connection is set up) in the resolution of microseconds (Figure 2), in addition to the UDP header information. We believe that this information is sufficient for most control algorithms.

Users can define their own control packets using the Type 2 information in the UDT control packet header (Figure 2). The detailed control information carried by these packets varies depending on the packet types. At the receiver side, users need to override processCustomMsg to tell UDT/CCC how to process these new types of packets.

$\begin{array}{lll}0 & 1\end{array}$

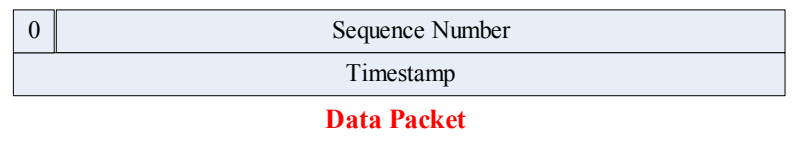

\begin{tabular}{|l|l|l|l|}
0 & \multicolumn{2}{c|}{15} \\
\hline 1 & Type 1 & Type 2 & ACK Sequence Number \\
\hline \multicolumn{3}{|c|}{ Control Information } \\
\hline
\end{tabular}

Control Packet

Figure 2. UDT Packet Header Structures. The first bit of the packet header is a flag to indicate if this is a data packet (0) or a control packet (1). Data packets contain a 31-bit sequence number and a 32-bit timestamp. In the control packet header, bit 1- 4 is the packet type (type 1) information. Type $0-6$ are used by UDT, whereas type 7 is used for user defined types, whose detail type information is put in bit $5-15$ (type 2). The detailed control information depends on the packet type.

Note that UDT's packet-based sequencing with the packet size information provided by UDP is equivalent to TCP's byte-based sequencing and can also support data streaming.

\subsubsection{Performance Monitoring}

Protocol performance information supports the decisions and diagnosis of a control algorithm. For example, certain algorithms need some history information to tune the future packet sending rate. Meanwhile, when testing new algorithms, performance statistics and internal protocol parameters are needed.

The performance monitor provides information including the duration time since the connection was started, RTT, sending rate, receiving rate, loss rate, packet sending period, congestion window size, flow window size, number of ACKs, and number of NAKs. UDT records these traces whenever the values are changed.

These performance traces can be read in three categories (when applicable): the aggregate values since the connection started, the local values since the last time the trace is queried, and the instant values when the query is made.

\subsection{The UDT Protocol}

UDT is an end-to-end, unicast, duplex, connection-oriented, streaming data transport protocol. 
Inside the UDT layer, there are two basic logical parts: the sender and the receiver. The sender is responsible for sending data packets according to congestion and flow control, whereas the receiver is responsible for receiving and processing packets, as well as sending control packets when necessary.

Figure 3 describes the relationship between the UDT sender and receiver. In Figure 3, the UDT entity A sends application data to the UDT entity B. The data is sent from A's sender to B's receiver, whereas the control flow is exchanged between the receivers.

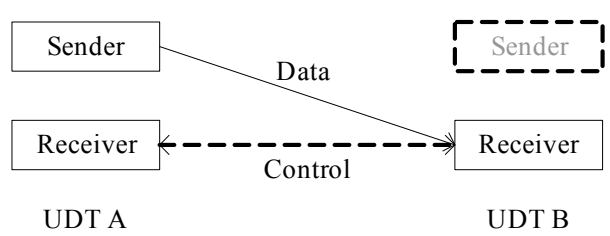

Figure 3. Relationship between UDT Sender and Receiver. All UDT entities have the same architectures, each having both a sender and receiver. This figure demonstrates the situation when a UDT entity $A$ sends data to another UDT entity B. Data is transferred from $A$ 's sender to $B$ 's receiver, whereas control information is exchanged between the receivers.

The detailed UDT protocol specification can be found in [42]. In the rest of the section, we will introduce the abstract UDT sending and receiving algorithms and how the control event handlers are processed in these algorithms. The detailed implementation will be introduced in next section.

\subsubsection{The Sending Algorithm}

Figure 4 describes the abstract sending algorithm. In this algorithm, a sender's loss list is a data structure that records the lost data packets when informed of them by loss reports from the receiver or by sender side timeouts. ACK and NAK are the abbreviations of acknowledgment and loss report (negative acknowledgment), respectively.

1) If there is no application data to send, sleep until it is activated by the application.

2) Packet sending:

a) If the sender's loss list is not empty and the number of unacknowledged packets does not exceed the congestion window size, remove the first lost sequence number from the list and pack the corresponding packet.

b) Otherwise, if the number of unacknowledged packets does not exceed the congestion and the flow window sizes, pack a new packet.

c) Otherwise, wait here until an ACK or NAK is received, or timeout occurs. Go to Step 1.

3) onPktSent().

4) Send the packed packet out.

5) Wait until the next packet sending time. Go to Step 1.

Figure 4. UDT Sending Algorithm.

Step 2.a and 2.b are the window/flow control, which limits the number of unacknowledged packets. Retransmissions (2.a) is sent first if there is any and only limited by congestion window.
Regular packet sending (2.b) is limited to either the congestion window size or the flow window size, whichever one is smallest.

Step 2.c implements self-clocking. In a pure window-based control protocol, the packet sending is blocked here until an ACK comes. Note that the timeout in this step is used to break the deadlock when there is no feedback. It is different from the packet sending timeout in the onTimeout method.

Step 5 is the rate control, which suspends the data sending until the next sending time. In a pure window-based control protocol, the inter-packet time is set to 0 , and thus this step is always skipped.

Both rate and window based approaches can be applied here. There are two specific situations. The first one is window-based control with pacing. In this situation an inter-packet time is calculated each time the congestion window size is updated, and Step 5 is used for the packet sending to sleep for this inter-packet time. The second situation is more complicated rate-based control with self-clocking. In the second situation, Step 5 is skipped, whereas Step 2.c is used for the packet sending to sleep for a certain time. Each time an ACK or a NAK is received, the sender compares the time passed since the last time a packet was sent and the expected inter-packet time, and none, one, or more packets (a burst) may be sent. A virtual congestion window may be used to control packet sending. This second method is like the one used in TFRC [9].

\subsubsection{The Receiving Algorithm}

Figure 5 describes the receiving algorithm. In this algorithm, the receiver's loss list is a data structure to store the sequence numbers of the lost packets. EXP is the abbreviation for timeout (expiration).

1) Query the timers

a) If ACK timer is expired and there are new packets to acknowledge, send back an ACK report; otherwise, if the user-defined ACK interval is reached, send back a lightweight ACK report.

b) If NAK timer is expired and the receiver's loss list is not empty, send back a NAK report;

c) If EXP timer is expired and there are sent but unacknowledged packets, execute onTimeOut(), and put the sequence numbers of these packets into the sender's loss list;

d) Reset the expired timers.

2) Start time bounded UDP receiving. If nothing is received before the UDP timer expires, go to Step 1.

3) If there is no unacknowledged packet, reset the EXP timer.

4) If the received packet is a control packet, process it, and reset EXP timer if it is an ACK or NAK; According to the packet type, one of the following callback functions may be executed: on $A C K()$; onLoss(); processCustomMsg(); Go to Step 1.

5) Process the data packet.

6) Check packet loss. If there are packet losses, insert the sequence numbers of the lost packets into the receiver's loss list and generate a loss report (NAK).

7) onPktReceived () ; Go to Step 1.

Figure 5. UDT Receiving Algorithm. 
The receiver uses self-clocked timers to trigger acknowledgment, loss reports, and timeout events (step 1 and 2). This timing mechanism takes advantage of time-bounded UDP receiving provided by OSes (e.g., using the SO_RCVTIMEO option).

In Step 1.c, UDT opts to retransmit all unacknowledged packets because it does not maintain per packet timeout timer due to efficiency reason.

As soon as a packet is received, the receiver processes the new packet according to its type (control packet in Step 4 and data packet in Step 5).

If there is no unacknowledged packet, the receiver simply resets the EXP timer (Step 3); otherwise, the EXP timer only resets when it is an ACK or NAK packet (Step 4).

Note that UDT has its own ACK and NAK processing mechanism in addition to the user-defined event handler for data reliability purposes.

Step 6 is to check packet loss. Various loss detection techniques such as robust reordering [38] can be used here. UDT uses packet based sequencing and the packet size can be determined from the UDP interface.

\section{IMPLEMENTATION}

One particular motivation of UDT/CCC is to support high performance data transfer in high-speed networks. Efficiency was always a major guideline when we implemented the UDT library. The efficiency of a protocol mainly depends on two factors: the congestion/flow control algorithm and the protocol design/implementation. UDT/CCC inherits much of its design and implementation from the UDT transport protocol [1]

Another important implementation objective is to provide good support for applications. User space libraries can be much easier to get deployed, but it is difficult for them to be transparent to applications. Therefore, existing applications may need to change their source codes. Our implementation makes a great effort to reduce the application developers' work.

\subsection{Software Architecture}

Figure 7 depicts the UDT/CCC software architecture. The UDT layer has five function components: the API module, the sender, the receiver, the listener, and the UDP channel, as well as four data components: sender's protocol buffer, receiver's protocol buffer, sender's loss list, and receiver's loss list.

Because UDT is bi-directional, all UDT entities have the same structure. The sender and receiver in Figure 6 have the same relationship as that in Figure 2.

The API module is responsible for interacting with applications. The data to be sent is passed to the sender's buffer and sent out by the sender into the UDP channel. At the other side of the connection (not shown in this figure but it has the same architecture), the receiver reads data from the UDP channel into the receiver's buffer, reorders the data, and checks packet losses. Applications can read the received data from the receiver's buffer.

The receiver also processes received control information. It will update the sender's loss list (when NAK is received) and the receiver's loss list (when loss is detected). Certain control events will trigger the receiver to update the congestion control module, which is in charge of the sender's packet sending.
The UDT socket options are passed to the sender/receiver (synchronization mode), the buffer management modules (buffer size), the UDP channel (UDP socket option), the listener (backlog), and CC (the congestion control algorithm). Options can also be read from these modules and provided to applications by the API module.

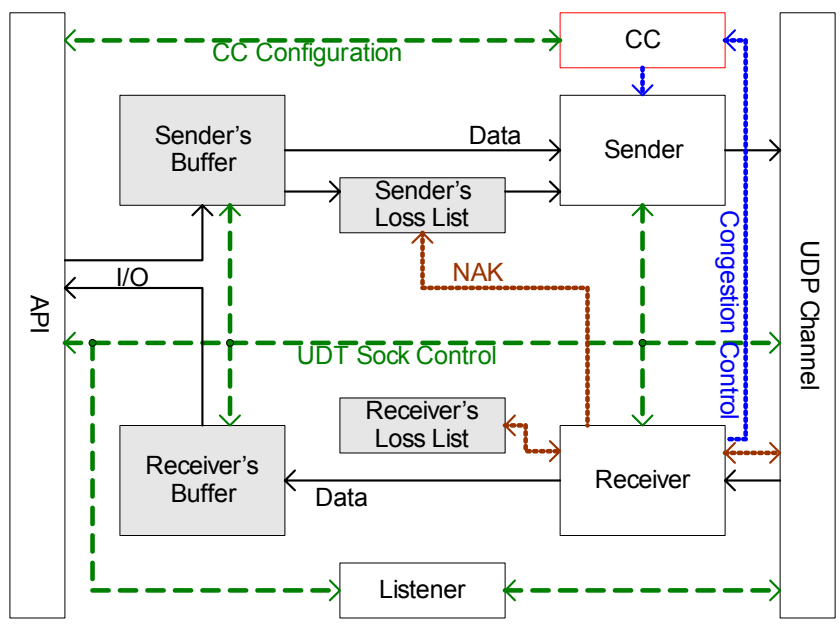

Figure 6. UDT/CCC Implementation. The solid line represents the data flow, and the dashed line represents the control flow. The shading blocks (buffers and loss lists) are the four data components, whereas the blank blocks (API, UDP channel, sender, receiver, and listener) are function components.

Since high-end workstations usually have multiple processors, UDT use a multi-threading implementation. The sender, the receiver, and the listener are concurrent threads, but they are only started when necessary (lazy start).

\subsection{Application Interface}

An application can make use of the UDT/CCC library in four ways. The library provides a set of $\mathrm{C}++$ API that is very similar to the system socket API. Network programmers can learn it easily and use it in a similar way as using TCP sockets. In particular, applications can use the setsockopt/getsockopt method to set and configure a specific congestion control algorithm at run time.

When used in applications written by languages other than $\mathrm{C} / \mathrm{C}++$, an API wrapper can be used. So far, both Java and Python UDT API wrappers have been developed.

Certain applications have a data transport middleware to make use of multiple transport protocols. In this situation, a new UDT driver can be added to this middleware, and then used by the applications transparently. For example, a UDT XIO driver has been developed so that the library can be used in Globus applications.

Finally, our library also provides a set of C API that has exactly the same semantics as the system socket API. An existing application can be re-compiled and linked against the UDT/CCC C library. In this way, the applications use our library transparently without any changes to the source codes. There is one limitation, though. UDT does not support multi-process models (e.g., using fork system call) due to efficiency 
considerations, so this method does not work if the existing application uses the same sockets in multiple processes.

\section{EVALUATION}

We already showed that UDT/CCC is easy to get deployed and requires little change in applications. We now focus on another two important characteristics to evaluate UDT/CCC: 1) the expressiveness (how many control algorithms can be implemented with UDT/CCC and how simply to implement them?) and 2) the similarity (how well can UDT/CCC based implementations simulate their native implementations?).

\subsection{Expressiveness}

To evaluate the expressiveness of UDT/CCC, we implement a set of representative control algorithms using the library. Any algorithms belonging to a similar set can be implemented in a similar way. Meanwhile, we show that the implementation is simple and easy to learn.

In this subsection, we describe in detail how to implement control algorithms of rate based UDP, TCP variants, including both lossbased and delay-based algorithms, and group transport protocols as well.

UDT/CCC uses an object-oriented design. It provides a base $\mathrm{C}++$ class (CCC) that contains all the functions and event handlers described in Section 2.2. A new control algorithm can inherit from this class and redefine certain control event handlers.

The implementation of any control algorithm is to update at least one of the two control parameters: the congestion window size ( $\left.m \_d C W n d S i z e\right)$ and the inter-packet time ( $m \_$dPacketPeriod) both of which are CCC class member variables.

\subsubsection{Rate-based UDP}

A rate-based reliable UDP library (CUDPBlast) is often used to transfer bulk data over private links. To implement this control mechanism, CUDPBlast initializes the congestion window with a very large value so that the window size will not limit the packet sending. The rest is to provide a method to assign a data transfer rate to a specific CUDPBlast instance. A piece of pseudo code is shown below:

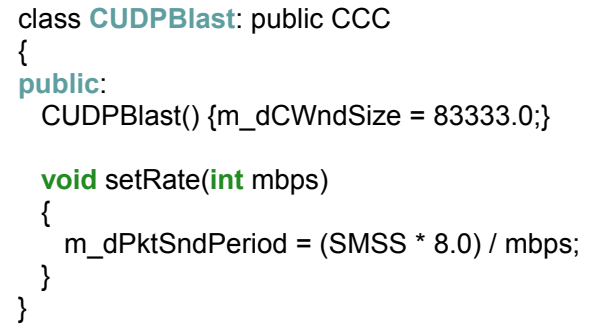

By using setsockopt an application can assign CUDPBlast to a UDT socket and by using getsockopt the application can obtain a pointer to the instance of CUDPBlast being used by the UDT socket. The application can then call the setRate method of this instance to set or modify a fixed sending rate at any time.

\subsubsection{Standard TCP (TCP NewReno)}

As a more complex example, we further show how to use the UDT/CCC library to implement the standard TCP congestion control algorithm (CTCP). Because a large portion of new proposed congestion control algorithms are TCP-based, this CTCP class can be further inherited and redefined to implement more TCP variants, which we will describe in the next two subsections.

TCP is a pure window-based control protocol. Therefore, during initialization, the inter-packet time is set to zero. In addition, TCP need data packets to be acknowledged frequently, usually every one or two packets ${ }^{1}$. This is also configured in the initialization.

TCP does not need explicit loss notification, but uses three duplicate ACKs to indicate packet loss. Therefore, for congestion control, CTCP only redefined two event handlers: onACK and onTimeout. In onACK, CTCP detects duplicate ACKs and takes proper actions. Here is the pseudo code of the fast retransmit and fast recovery algorithm in RFC 2581:

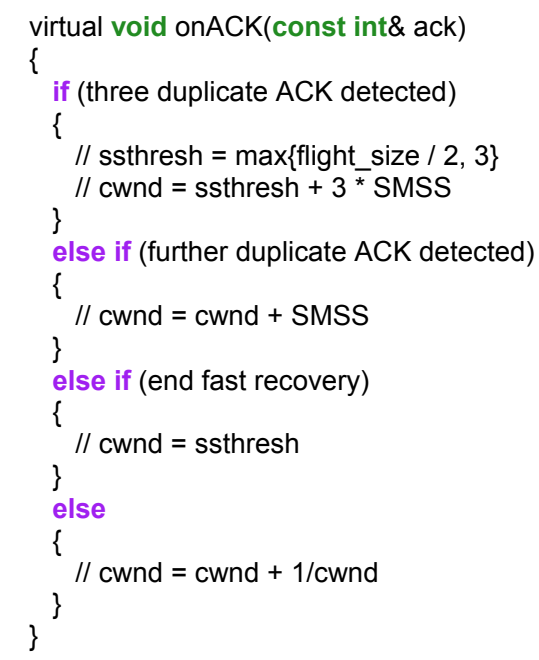

The CTCP implementation can provide more TCP event handlers such as DupACKAction and ACKAction, which will further reduce the work of implementing new TCP variants.

Note that here we are only implementing TCP's congestion control algorithm, but NOT the whole TCP protocol. The UDT/CCC library does not implement exactly the same protocol mechanisms as in the TCP specification but it does provide similar functionality. For example, TCP uses byte-based sequencing whereas UDT uses packet-based sequencing, but this should not prevent CTCP from simulating TCP's congestion avoidance behavior.

\subsubsection{New TCP Algorithms (Loss-based)}

New TCP variants that use loss-based approaches usually redefine the increase and decrease formulas of the congestion window size. Implementations of these protocols can simply inherit from CTCP and redefine proper TCP event handlers.

For example, to implement Scalable TCP, we can simply derive a new class from CTCP, and override the actions of increasing (by

1 Although TCP uses accumulative acknowledgements, a TCP implementation usually acknowledges at the boundary of a data segment. This is equivalent to acknowledging a UDT data packet in CTCP. 
0.1 instead of $1 /$ cwnd) and decreasing (by $1 / 8$ instead of $1 / 2$ ) the congestion window size.

Similarly, we have also implemented HighSpeed TCP (CHS), BiC TCP (CBiC), and TCP Westwood (CWestwood).

\subsubsection{New TCP Algorithms (Delay-based)}

Delay-based algorithms usually need accurate timing information for each packet. For efficiency, UDT does not calculate RTT for each data packet because it is unnecessary for most control algorithms. However, this can be done by overriding onPktSent and $o n A C K$ event handlers, where the time of packet sending and the arrival of its acknowledgement can be recorded. For algorithms preferring one-way delay (OWD) information, each UDT packets contains the sending time in its packet header, and a new algorithm can override onPktReceived to calculate OWD.

Using the strategy described above, we implement the TCP Vegas (CVegas) control algorithm. CVegas uses its own data structure to record packet departure timestamps and ACK arrival timestamps, and then calculates accurate RTT values. With simple modifications to the control formulas, we further implement FAST TCP (CFAST).

\subsubsection{Group Transport Control}

While we have demonstrated that UDT/CCC can be used to implement end-to-end unicast congestion control algorithms, we now show that it can also be used to implement group-based control mechanisms, such as CM and GTP.

To support this feature, the new algorithm class simply needs to implement a central manager to control a group of connections. The control parameters are calculated by the central manager and then fed back to the control class instance of each individual connection.

We implemented GTP (CGTP) as an example of group-based control mechanisms. The GTP protocol controls a group of flows with the same destination. CGTP tunes the packet sending rate at the receiver side periodically and feeds back the parameters using UDT/CCC's sendCustomMsg method.

\subsubsection{Summary}

We have implemented nine example algorithms using UDT/CCC, including rate-based reliable UDP, TCP and its variants, and group-based protocols. We demonstrated that our UDT/CCC library can support a large variety of congestion control algorithms, which are supported by only 8 event handlers, 4 protocol control functions, and 1 performance monitoring function.

The concise UDT/CCC API is easy to learn. In fact, it takes a small piece of code to implement most the algorithms described above. Table 1 lists the lines of code (LOC) of implementations of TCP algorithms using UDT/CCC, as well as the LOC of those native implementations (Linux kernel patches). The LOC value is estimated by the number of semicolons in the corresponding $\mathrm{C} / \mathrm{C}++$ code segment.

To give more insight into the difference between LOCs in UDT/CCC based implementations and native implementations, we use the FAST TCP case as an example. The 31 lines of CFAST only implement the FAST congestion avoidance algorithm, whereas much of its codes, especially the timing part, are inherited from CVegas. In contrast, of the 367 lines of FAST
TCP patch, 142 of them are used to implement the FAST protocol (new files), 81 lines are used to modify the Linux TCP files, 86 lines are used to do monitoring and statistics, and 58 lines are used to do burst control and pacing.

Table 1. Lines of Code (LOC) of implementations of TCP algorithms. This table lists LOC of different TCP algorithms implemented using UDT/CCC and their respective Linux kernel patches (native implementations). The LOC of Linux patches include both added lines and removed lines.

\begin{tabular}{|c|c|c|c|}
\hline \multirow{2}{*}{ Protocol } & \multirow{2}{*}{ UDT/CCC } & \multicolumn{2}{|c|}{ Native } \\
\hline & & Added & Removed \\
\hline TCP & 28 & - & \\
\hline Scalable TCP & 11 & 192 & 29 \\
\hline HighSpeed TCP & 8 & 27 & 1 \\
\hline $\mathrm{BiC}$ TCP & 38 & 248 & 30 \\
\hline TCP Westwood & 27 & 145 & 2 \\
\hline TCP Vegas & $37+36^{2}$ & 132 & 6 \\
\hline FAST TCP & 31 & 365 & 2 \\
\hline
\end{tabular}

As a reference point, the UDT library has 3134 lines of effective code (i.e., excluding comments, blank lines, etc.), SABUL has 2670 lines of code, and the RBUDP library has approximately 2330 lines of code. While these numbers are not enough to reflect the complexity of implementing a transport protocol, the much smaller number of LOC values of UDT/CCC based implementation can indicate the simplicity of using UDT/CCC.

The class inheritance relationship of these UDT/CCC implemented algorithms can be found in Figure 7. Code reuse by class inheritance also contributes to the small LOC values of those TCP-based algorithms.

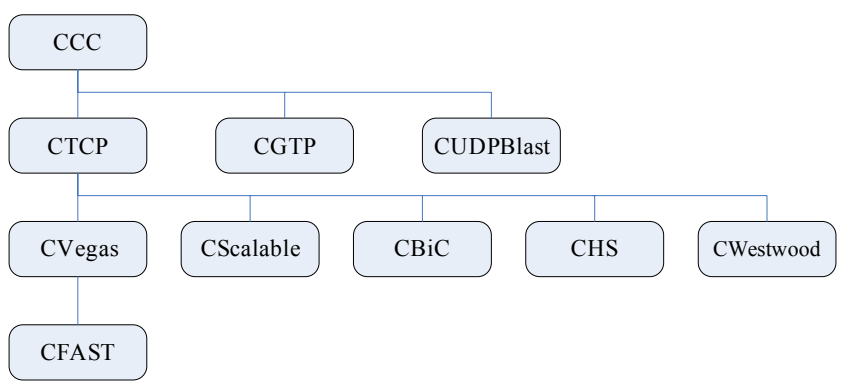

Figure 7. UDT/CCC based protocols. This figure shows the class inheritance relationship among the control algorithms we implemented. Note that this is only for the purpose of code reuse, but NOT imply any other relationship among these algorithms.

\subsection{Similarity}

It is a fundamental goal for UDT/CCC to simulate the performance of a control algorithm's native implementation or realize the algorithm's theoretical performance.

In most cases, congestion/flow control algorithms are the most significant factor that determines a protocol's performance-related behavior (throughput, fairness, and stability). Less significant

\footnotetext{
${ }^{2}$ CVegas reuses a timing class implemented by UDT, which contains 36 lines of code.
} 
factors include other protocol control mechanisms, such as RTT calculation, timeout calculation, acknowledgment interval, etc.

We have made most of these control mechanisms configurable through the CCC interface and the UDT protocol control interface. In this subsection we will show that a UDT/CCC based implementation demonstrates similar performance to its native counterpart.

Since TCP is probably the most representative congestion control protocol, we compared an application level TCP implementation using our UDT/CCC library (CTCP) against the standard TCP implementation provided by Linux kernel 2.4.18.

The experiment was performed between two Linux boxes between Chicago and Amsterdam. The link is $1 \mathrm{~Gb} / \mathrm{s}$ with $110 \mathrm{~ms}$ RTT and was reserved for our experiment only in order to eliminate cross traffic noises. Each Linux box has dual Xeon 2.4GHz processors and was installed with Linux kernel 2.4.18. We started multiple TCP and CTCP flows in separate runs, each of which was kept running for at least 60 minutes. The total TCP buffer size was set to at least the size of BDP (bandwidth delay product). Both TCP and CTCP experiments used the same testing program (except the connections were TCP and CTCP, respectively) with same configuration (buffer size, etc.).

We recorded the aggregate throughput (value between 0 and 1000 Mbps), fairness index (value between 0 and 1), and stability index (equal to or greater than 0 ) in Table 2. The definitions of the fairness index and stability index can be found in $[1,26]$. The fairness index represents how fairly the bandwidth is shared by concurrent flows and larger values are better. The stability index describes the oscillations of the flows and smaller values mean less oscillation. These three measurements summarize the major performance characteristics of a congestion control algorithm.

Table 2. Performance characteristics of TCP and CTCP with various parallel flows. The table lists the aggregate throughput (in $\mathrm{Mb} / \mathrm{s}$ ), fairness index, and stability index of concurrent TCP and CTCP flows. Each row records an independent run with a different number of parallel flows.

\begin{tabular}{|l|l|l|l|l|l|l|}
\hline \multirow{2}{*}{$\begin{array}{c}\text { Flow } \\
\text { \# }\end{array}$} & \multicolumn{2}{|c|}{ Throughput } & \multicolumn{2}{c|}{ Fairness } & \multicolumn{2}{c|}{ Stability } \\
\cline { 2 - 7 } & TCP & CTCP & TCP & CTCP & TCP & CTCP \\
\hline 1 & 112 & 122 & 1 & 1 & 0.517 & 0.415 \\
\hline 2 & 191 & 208 & 0.997 & 0.999 & 0.476 & 0.426 \\
\hline 4 & 322 & 323 & 0.949 & 0.999 & 0.484 & 0.492 \\
\hline 8 & 378 & 422 & 0.971 & 0.999 & 0.633 & 0.550 \\
\hline 16 & 672 & 642 & 0.958 & 0.985 & 0.502 & 0.482 \\
\hline 32 & 877 & 799 & 0.988 & 0.997 & 0.491 & 0.470 \\
\hline 64 & 921 & 716 & 0.994 & 0.996 & 0.569 & 0.529 \\
\hline
\end{tabular}

From Table 2, we find that TCP and CTCP have pretty similar throughput for small numbers of parallel flows. However, as the number of parallelism increases, CTCP stops increasing its throughput first and thus has a significantly smaller throughput than TCP when there are 64 parallel flows ${ }^{3}$. Further analysis indicates that the reason for this is that CTCP costs more CPU

3 TCP throughput will also start to decrease as the number of parallel flows increases [12] because of the system overhead and the global synchronization phenomenon. than kernel implemented TCP and with 64 flows the CPU time has been used up ${ }^{4}$. To verify this assertion, we started another experiment using machines with dual AMD 64-bit Opteron processors and this time CTCP reaches more than $900 \mathrm{Mbps}$ at 64 parallel flows. In fact, according to the CPU usage we traced in the experiment in Table 2, a CTCP sender uses $60-100 \%$ more CPU time than a TCP sender, whereas a CTCP receiver uses $10-$ $30 \%$ more CPU time than a TCP receiver.

In spite of the CPU utilization limitation, both of the implementations have similar performance on fairness and stability. They both realize good fairness with near-one fairness indexes, as the AIMD algorithm indicates. The stability indexes are around 0.5 for all runs.

We also note that CTCP has slightly better performance than TCP under small concurrencies. We believe that this is acceptable experimental error: differences between CTCP and TCP (experiment time, UDP vs. TCP traffic, user space vs. kernel space) may cause them to experience different packet loss rates.

Although the CPU overhead of UDT/CCC may limit its usage in certain scenarios, we argue that the library is still useful in many other situations. First, in high performance computing, there are usually only a small number of flows sharing the high bandwidth. In this case, the threading overhead is low. Second, the overhead can be overcome by more powerful processors and more machines. Third, the overhead of UDT/CCC only exists when compared to kernel space implementations; it is insignificant when compared to other user space implementations. Finally, profiling analysis indicates that the other major overhead of CTCP comes from the acknowledging, control mechanisms with less frequent acknowledging would result in much less overhead.

We are, however, quite aware of the importance of CPU efficiency. Our current work is focused on code optimization.

We have also performed the same experiments on similarity and performance on other systems with different operating systems (Linux, BSD, OS X, Windows XP), hardware (Intel, AMD, and PowerPC processors), and networks. Although specific systems have more or less impact on the results, all the experiments conform to the similarity and performance trends we obtained from the experiments described in this section.

In addition to the experiments above, we have also tested several reliable UDP-based protocols such as UDP Blast (CUDPBlast) to examine if the UDT/CCC based implementation conforms to the protocol's theoretical performance. (In the case of UDP Blast, the expected throughput is the fixed value set by applications.) We also examined the performance of UDT/CCC in a real streaming merge application, in which the receiver (where data is merged) requests an explicit sending rate to the data sources. This service is provided by a specific control mechanism implemented using UDT/CCC.

\footnotetext{
${ }^{4}$ CTCP starts user level threads to support multiplexing. The number of threads is proportional to the number of parallel flows. These threads and the synchronization among them bring CPU overheads, which increase as the number of parallel flows increases.
} 


\section{RELATED WORK}

There are few user level protocol stacks that provide a programming interface for user-defined congestion control algorithms as UDT/CCC does.

The Globus XIO [39] library has somewhat similar objectives, but the approach is quite different. XIO implements a set of primitive protocol components and APIs for fast creation or prototyping new protocols, which helps simply the lower level simplification such as timing and message passing. In contrast, UDT/CCC allows users to focus only on the congestion control algorithm, and thus usually results a much smaller program.

Less similar user level libraries include several user level TCP implementations $[15,16,17,18,19]$. One particular implementation is the Alpine [15] library. Alpine is an attempt to move the entire kernel protocol stack into the user space, and provides (almost) transparent application interfaces at the same time. None of these libraries provide programmable interfaces.

In kernel space, the most similar work to UDT/CCC is probably the icTCP [4] library. It exposes key TCP parameters and provides controls to these parameters to allow new TCP algorithms deployed in user space. Despite the different nature of kernel and user space implementations, icTCP limits the update on TCP controls only, whereas UDT/CCC supports a broader set of protocols. Other work that uses a similar approach to icTCP includes Web100/Net100 [6] and CM [5].

Another work, STP [3], has more radical changes but also has more powerful expression ability. The STP's approach is to provide a set of protocol implementation APIs in a sandbox. Meanwhile, STP itself is a protocol that supports run time code upgrading; thus, new protocols or algorithms can be deployed implicitly. To address the security problem arising from untrusted code, STP involves a complex security mechanism.

Yet another more complex library is the $x$-kernel [13]. $x$-kernel is an OS kernel designed to support data transport protocol implementations. The support mechanism of $x$-kernel is a modular based system and it is more decomposed than STP. Besides the support of protocol implementation, $x$-kernel has many optimizations inside the OS kernel for data communications.

Other modularized approaches include Horus [14], CTP [22] and its high performance successor [23].

While some of these in-kernel libraries may have performance and transparency advantages, their goals of fast deployment of new protocols/algorithms are compromised by the difficulty of getting themselves deployed. For example, $x$-kernel has been proposed for more than a decade and it still remains a research tool. In contrast, UDT/CCC library provides a very practical solution for the time being.

In addition, kernel space approaches need to protect their host systems and the network from security problems and they have to limit users' privileges to control the protocol behavior. For example, both STP and icTCP prevent new algorithms from utilizing more bandwidth than standard TCP. Such limitations are improper to the new control algorithms for high-speed networks such as Scalable, HighSpeed, BiC, and FAST. The security problem is much less serious for UDT/CCC because it is at user space and it is only installed as needed (in contrast, those libraries such as icTCP and STP will be accessible to every user if they are accepted by OS vendors).

Finally, there is another category of related work that attempts to provide a protocol language for easier, faster, or more readable protocol implementation. Such work includes Prolac [20] and FoxNet [21].

\section{CONCLUSION}

The maturity of high-speed wide area networks encouraged the emergence of numerous new applications, and new control mechanisms supporting these applications as well. It has often been the case that implementing these new control algorithms in the kernel is not practical or proper. On the one hand, the wide deployment of new protocols or algorithms usually suffers long time lag. On the other hand, OS vendors may only choose a very small number of protocols to implement in kernel.

We have presented a user level transport protocol stack named UDT/CCC, which allows user defined congestion control algorithms to be easily implemented. Our UDT/CCC library enables easy implementations of a large variety of control algorithms while these implementations can still match the performance characteristics of those native implementations.

However, UDT/CCC is not meant to replace kernel protocol stacks or proposed as a mean to implement any new protocols. Instead, it provides a practical alternative when a kernel space approach is difficult to implement, evaluate, and deploy. These use scenarios include the implementation of a new application or network specific congestion mechanism and the evaluation of new congestion control algorithms.

Finally, we are aware of the CPU utilization limitations of the current UDT/CCC implementation. While there are unavoidable efficiency side effects for application level protocol implementations because of the additional memory copy and context switches, profiling analysis shows that there is still space to further optimize the implementation.

The UDT project, including the feature described in this paper, is meant for productivity use, rather than just for research or prototyping. The UDT library is open source software and can be obtained from http://udt.sf.net.

\section{ACKNOWLEDGMENTS}

This work was supported in part by the Department of Energy under grant DE-FG02-04ER25639, the U.S. Army Pantheon Project, and the National Science Foundation under grant ANI9977868 and SCI-0430781.

We would also like to thank the five anonymous reviewers for their useful and constructive suggestions.

\section{REFERENCES}

[1] Yunhong Gu, Xinwei Hong, and Robert Grossman: Experiences in Design and Implementation of a High Performance Transport Protocol, SC 2004, Nov 6 - 12, Pittsburgh, PA, USA.

[2] W. Feng and P. Tinnakornsrisuphap: The Failure of TCP in High-Performance Computational Grids, SC 2000, Dallas, TX, Nov. 00. 
[3] Parveen Patel, Andrew Whitaker, David Wetherall, Jay Lepreau, and Tim Stack: Upgrading Transport Protocols using Untrusted Mobile Code, in Proceedings of the 19th ACM Symposium on Operating System Principles, October 19-22, 2003.

[4] Haryadi S. Gunawi, Andrea C. Arpaci-Dusseau, Remzi H. Arpaci-Dusseau: Deploying Safe User-Level Network Services with icTCP, OSDI 2004.

[5] David G. Andersen, Deepak Bansal, Dorothy Curtis, Srinivasan Seshan, and Hari Balakrishnan: System Support for Bandwidth Management and Content Adaptation in Internet Applications, Proc. 4th USENIX Conference on Operating Systems Design and Implementation (OSDI 2000), San Diego, CA, October 2000.

[6] M. Mathis, J. Heffner, and R. Reddy: Web100: Extended TCP Instrumentation for Research, Education and Diagnosis, ACM Computer Communications Review, Vol 33, Num 3, July 2003.

[7] R. R. Stewart, Q. Xie, K. Morneault, C. Sharp, H. J. Schwarzbauer, T. Taylor, I. Rytina, M. Kalla, L. Zhang, and V. Paxson: Stream control transmission protocol. RFC 2960, Oct. 2000.

[8] Eddie Kohler, Mark Handley, Sally Floyd, Jitendra Padhye: Datagram Congestion Control Protocol (DCCP), http://www.icir.org/kohler/dcp/. Jan. 2005.

[9] Sally Floyd, Mark Handley, Jitendra Padhye, and Joerg Widmer: Equation-Based Congestion Control for Unicast Applications, ACM SIGCOMM 2000, Stockholm, Aug. 2000.

[10] M. Allman, V. Paxson, W. Stevens, TCP Congestion Control, IETF, RFC 2581, April 1999.

[11] V. Paxson and M. Allman: Computing TCP's Retransmission Timer, RFC 2988, IETF, Nov. 2000.

[12] Harimath Sivakumar, Stuart Bailey, Robert L. Grossman. PSockets: The Case for Application-level Network Striping for Data Intensive Applications using High Speed Wide Area Networks, SC 2000, Dallas, TX, Nov. 2000.

[13] N. C. Hutchinson and L. L. Peterson: The x-Kernel: An architecture for implementing network protocols, IEEE Transactions on Software Engineering, 17(1): 64-76, Jan. 1991.

[14] R. van Renesse, K. P. Birman, R. Friedman, M. Hayden, and D. A. Karr: A framework for protocol composition in Horus, in Proceedings of the Fourteenth Annual ACM Symposium on Principles of Distributed Computing, pages 80-89, Ottawa, Ontario, Canada, 2-23 Aug. 1995.

[15] David Ely, Stefan Savage, and David Wetherall: Alpine: A user-level infrastructure for network protocol development, in Proc. 3rd USENIX Symposium on Internet Technologies and Systems (USITS 2001), pages 171-183, March 2001.

[16] Thekkath, C. A., Nguyen, T. D., Moy, E., and Lazowska, E. D: Implementing network protocols at user level, IEEE/ACM Transactions on Networking, 1(5): 554--565, October 1993.

[17] A. Edwards and S. Muir: Experiences Implementing A HighPerformance TCP In User-Space, in Proc. ACM SIGCOMM 1995, Cambridge, MA, pages 196 - 205.
[18] Prashant Pradhan, Srikanth Kandula, Wen Xu, Anees Shaikh, Erich Nahum: Daytona: A User-Level TCP Stack, http://nms.lcs.mit.edu/ kandula/data/daytona.pdf.

[19] Kieran Mansley: Engineering a user-level TCP for the CLAN network, in SIGCOMM 2003 workshop on Network-I/O convergence: experience, lessons, implications.

[20] E. Kohler, M. F. Kaashoek, and D. R. Montgomery: $A$ Readable TCP in the Prolac Protocol Language, in Proceedings of SIGCOMM '99, pages 3-13, Cambridge, Massachusetts, Aug. 1999.

[21] Edoardo Biagioni: A structured TCP in Standard ML, in Proceedings of the ACM SIGCOMM Conference on Communications Architectures, Protocols and Applications, pages 36-45, London, England, 1994.

[22] Gary T. Wong, Matti A. Hiltunen, and Richard D. Schlichting: A configurable and extensible transport protocol, IEEE Infocom 2001, April 22-26, 2001. Anchorage, Alaska, April 2001.

[23] Xinran Wu, Andrew A. Chien, Matti A. Hiltunen, Richard D. Schlichting and Subhabrata Sen, High Performance Configurable Transport Protocol for Grid Computing, in Proceedings of the 5th IEEE/ACM International Symposium on Cluster Computing and the Grid (CCGrid 2005).

[24] M. Gerla, M. Y. Sanadidi, R. Wang, A. Zanella, C. Casetti, and S. Mascolo. TCP Westwood: Congestion Window Control Using Bandwidth Estimation. IEEE Globecom 2001, Volume: 3, pp 1698-1702.

[25] L. Brakmo and L. Peterson. TCP Vegas: End-to-End Congestion Avoidance on a Global Internet. IEEE Journal on Selected Areas in Communication, Vol 13, No. 8 (October 1995) pages 1465-1480.

[26] C. Jin, D. X. Wei, and S. H. Low. FAST TCP: motivation, architecture, algorithms, performance. IEEE Infocom '04, Hongkong, China, Mar. 2004.

[27] S. Floyd. HighSpeed TCP for Large Congestion Windows. RFC 3649, Experimental Standard, Dec. 2003.

[28] T. Kelly. Scalable TCP: Improving Performance in Highspeed Wide Area Networks. ACM Computer Communication Review, Apr. 2003.

[29] L. Xu, K. Harfoush, and I. Rhee. Binary Increase Congestion Control for Fast Long-Distance Networks. IEEE Infocom '04, Hongkong, China, Mar. 2004.

[30] Y. Gu and R. L. Grossman: SABUL: A Transport Protocol for Grid Computing. Journal of Grid Computing. 2003, Volume 1, Issue 4, pp. 377-386.

[31] E. He, J. Leigh, O. Yu, T. A. DeFanti: Reliable Blast UDP: Predictable High Performance Bulk Data Transfer, IEEE Cluster Computing 2002, Chicago, IL 09/01/2002.

[32] C. Xiong, Leigh, J., He, E., Vishwanath, V., Murata, T., Renambot, L., DeFanti, T.: LambdaStream - a Data Transport Protocol for Streaming Network-intensive Applications over Photonic Networks, Third International Workshop on Protocols for Long-Distance Networks (PFLDnet 2005), Lyon, France, Feb. 2005. 
[33] M. Veeraraghavan, X. Zheng, H. Lee, M. Gardner, W. Feng, CHEETAH: Circuit-switched High-speed End-to-End Transport ArcHitecture, Proc. of Opticomm 2003, Oct. 1317, 2003. Dallas, TX.

[34] A. Szalay, J. Gray, A. Thakar, P. Kuntz, T. Malik, J. Raddick, C. Stoughton. J. Vandenberg: The SDSS SkyServer - Public Access to the Sloan Digital Sky Server Data, ACM SIGMOD 2002.

[35] Naveen Krishnaprasad, Venkatram Vishwanath, Shalini Venkataraman, Arun G.Rao, Luc Renambot, Jason Leigh, Andrew E.Johnson: JuxtaView - A Tool for Interactive Visualization of Large Imagery on Scalable Tiled Displays, in the proceedings of IEEE Cluster 2004, San Diego, CA, September 20-23, 2004.

[36] Ryan Wu and Andrew Chien: GTP: Group Transport Protocol for Lambda-Grids, in Proceedings of the 4th IEEE/ACM International Symposium on Cluster Computing and the Grid (CCGrid2004), Chicago, Illinois, April 2004.
[37] Mathis, M., Mahdavi, J., Floyd, S., and Romanow, A., TCP Selective Acknowledgement Options. RFC 2018, April 1996.

[38] Zhang, M., Karp, B., Floyd, S., and Peterson, L., RR-TCP: A Reordering-Robust TCP with DSACK, in Proceedings of the Eleventh IEEE International Conference on Networking Protocols (ICNP 2003), Atlanta, GA, November, 2003.

[39] Globus XIO: http://wwwunix.globus.org/toolkit/docs/3.2/xio/index.html. Retrieved on Apr. 3, 2005.

[40] E-VLBI, http://web.haystack.edu/e-vlbi/evlbi.html. Retrieved on Apr. 6, 2005.

[41] Qishi Wu, Nageswara S. V. Rao, Protocol for High-Speed Data Transport Over Dedicated Channels, Third International Workshop on Protocols for Long-Distance Networks (PFLDnet 2005), Lyon, France, Feb. 2005.

[42] Yunhong Gu and Robert L. Grossman, UDT: A Transport Protocol for Data Intensive Applications. Internet Draft. Work in progress. 